\title{
Évaluation du rapport bénéfice-risque de la suspension des anti-TNF alpha en chirurgie orale
}

\author{
Virginie Pannérec ${ }^{1}$, Sylvain Catros ${ }^{1,2,3,{ }^{*}, \text { Jean-Christophe Fricain }}{ }^{1,2,3}$ \\ 1 CHU de Bordeaux, Pôle d'Odontologie et de Santé buccale, Bordeaux, France \\ 2 Universté de Bordeaux, Bioingénierie tissulaire, U1026, Bordeaux, France \\ 3 INSERM, Bioingénierie tissulaire, U1026, Bordeaux, France
}

(Reçu le 13 septembre 2013, accepté le 30 mars 2014)

Mots clés : polyarthrite rhumatoïde / anti-TNF alpha / chirurgie orale / infection / retard de cicatrisation

Key words: rheumatoid arthritis / anti-TNF alpha / oral surgery / infection / wound healing delay

\begin{abstract}
Résumé - Introduction : Les anti-TNF alpha sont des médicaments immunomodulateurs. La polyarthrite rhumatoïde (PR) est la principale indication des anti-TNF alpha. Les sociétés savantes recommandent actuellement de suspendre les anti-TNF en cas de chirurgie mais cela pourrait présenter un risque de poussée de la PR. L'objectif de cette étude était d'établir le rapport bénéfice-risque de la suspension des anti-TNF en chirurgie orale. Méthodes : Une revue systématique de la littérature a été réalisée entre janvier 2003 et avril 2013. Résultats : 257 articles ont été analysés et 27 articles ont été retenus. La majorité des études concluaient à une absence d'augmentation des infections postopératoires pour les patients sous anti-TNF, sans pouvoir conclure de façon définitive. Concernant le risque de complications cicatricielles, la majorité des études ont montré des taux de complications similaires avec ou sans anti-TNF. Enfin, trois études ont rapporté des cas de poussée de PR après suspension de l'étanercept. Discussion : Le rapport bénéfice-risque de l'arrêt des anti-TNF chez les patients traités pour une PR doit être évalué au cas par cas. Des études avec un niveau de preuve plus élevé sont nécessaires pour conclure sur le rapport bénéfice-risque de l'arrêt des anti-TNF avant une intervention de chirurgie orale.
\end{abstract}

\begin{abstract}
Evaluation of the benefit/risk ratio of discontinuing anti-TNF therapy for oral surgery procedures. Introduction: Anti-TNF alpha are immunomodulatory therapies mainly employed for rheumatoid arthritis (RA). Several scientific societies have recommended interrupting anti-TNF alpha when surgery is planned. However, this interruption may induce a relapse of RA. The aim of this work was to evaluate the benefit / risk ratio related to the interruption or maintenance of anti-TNF therapy before oral surgery procedures. Methods: A systematic literature review including articles published from January 2003 to April 2013 was performed. Specific inclusion and exclusion criteria were determined. Results: 257 articles were analyzed and 27 articles were included in this literature review. Most of the studies did not find an increase in post-surgical infectious complications in patients taking anti-TNF. However, contradictory results were found in some studies and there were insufficient available data to conclude. Concerning delayed healing complications, most of the studies showed similar levels of complications. Finally, three studies described cases of RA relapse when Etanercept ${ }^{\circledR}$ was suspended. Discussion: The benefit/risk ratio of interrupting anti-TNF in patients treated for RA must be evaluated individually. It is necessary to perform studies containing a higher level of evidence before establishing new recommendations.
\end{abstract}

\section{Introduction}

Le TNF alpha (Tumor Necrosis Factor) est une cytokine ubiquitaire possédant des propriétés inflammatoires et antibactériennes. Elle est principalement sécrétée par les cellules du système réticulo-endothélial sous la forme d'un précurseur, le
« pro-TNF ». Sa forme définitive est produite sous l'effet d'une métalloprotéase présente au sein des cellules cancéreuses, bactériennes, ou virales. La plupart des cellules de l'organisme possèdent des récepteurs au TNF alpha. Cela explique les multiples actions directes ou indirectes de cette cytokine. Elle est impliquée dans de nombreuses pathologies inflammatoires,

\footnotetext{
*Correspondance : sylvain.catros@u-bordeaux.fr
} 
néoplasiques, vasculaires, infectieuses et auto-immunes comme la polyarthrite rhumatoïde (PR) [1]. La PR est caractérisée par la prolifération pseudo-tumorale du tissu synovial, aboutissant à une destruction des structures articulaires. La PR se complique de déformations articulaires, responsables dans $20 \%$ des cas d'une incapacité fonctionnelle.

Les anti-TNF sont classés en deux groupes : des anticorps monoclonaux (infliximab (IFX) ou Rémicade ${ }^{\circledR}$, adalimumab ou Humira ${ }^{\circledR}$, golimumab ou Simponi ${ }^{\circledR}$, certolizumab ou Cimzia ${ }^{\circledR}$ ) qui bloquent des protéines effectrices, et des récepteurs solubles (étanercept (ENT) ou Enbrel ${ }^{\circledR}$ ) qui miment l'action de récepteurs spécifiques. Chaque anti-TNF alpha a une pharmacocinétique spécifique. Leur activité est très ciblée et leur action conduit à une immunomodulation. Les anti-TNF ont pour principale indication la PR qui est l'une des maladies auto-immunes les plus fréquentes. Ces biothérapies très coûteuses sont prescrites actuellement en seconde intention, en cas de mauvais pronostic (PR réfractaire ou agressive) [2].

Les patients atteints de PR développeraient plus de parodontites [3, 4]. Ils perdraient leurs dents précocement, ce qui altèrerait directement leur qualité de vie [5]. Les sociétés savantes de rhumatologie préconisent actuellement la suspension de ce traitement immunomodulateur en cas de chirurgie programmée. En 2012, la Société française de chirurgie orale (SFC0) recommandait de contacter le médecin prescripteur et de suspendre le traitement dans des délais déterminés arbitrairement selon le type de molécule [6]. Cet arrêt pourrait être un risque de poussée de la maladie, associé à un risque infectieux en réponse aux corticoïdes prescrits en substitution des anti-TNF pour contrôler l'évolution de la PR [7].

L'objectif de cette étude était d'établir le rapport bénéficerisque que représente la suspension des anti-TNF alpha dans le cadre de la chirurgie orale.

\section{Matériels et méthodes}

\section{Schéma de l'étude}

Il s'agit d'une revue systématique de la littérature qui a été réalisée à partir des bases de données Medline (littérature internationale), Pascal (littérature francophone), Scopus (base de données transdisciplinaire), Google, et des sites Internet de sociétés savantes (recommandations).

\section{Définition des mots clés}

La définition des mots clés a été le plus large possible en utilisant le MeSH. Les mots clés ou association de mots clés utilisés dans les moteurs de recherche étaient: "tumor necrosis factors", "antagonists and inhibitors", "anti-TNF", "TNF alpha antagonist", "tumor necrosis factor inhibitors", "tumor necrosis factor antagonist", "tumor necrosis factor blockers", "practice guideline”, "practice guidelines", "guideline", "guidelines", "recommendations", "consensus development conference", "consensus development conferences", "health planning guidelines", "infection", "surgery", "rheumatoid arthritis", "remission induction" et "healing wound".

\section{Stratégie de recherche}

À partir des premières données recueillies, trois axes d'étude ont été définis, puis nous avons utilisé les combinaisons de mots clés suivantes pour chaque axe d'étude.

Évaluation du risque de complications infectieuses postchirurgicales des anti-TNF alpha

"tumor necrosis factors/antagonists and inhibitors" [MeSH] OR "anti-TNF" OR "TNF alpha antagonist" OR "tumor necrosis factor inhibitors" OR "tumor necrosis factor antagonist" OR "tumor necrosis factor blocker".

"practice guideline/pt" OR "practice guidelines as topic/

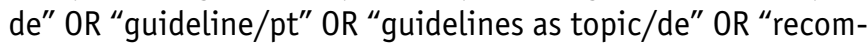
mendations" OR "consensus development conference" OR "consensus development conferences" $O R$ "health planning guidelines" [MeSH] OR "guideline, health planning" [MeSH] OR "recommendations, health planning".

"infection" [MeSH] AND "surgery" [MeSH] AND "rheumatoid arthritis" [MeSH].

\section{Impact des anti-TNF sur la cicatrisation}

"tumor necrosis factors/antagonists and inhibitors" [MeSH] OR "anti-TNF" OR "TNF alpha antagonist" OR "tumor necrosis factor inhibitors" OR "tumor necrosis factor antagonist" OR "tumor necrosis factor blocker".

"rheumatoid arthritis" [MeSH] AND "healing", "wound" [MeSH] "induction".

Risque de poussée et suspension des anti-TNF alpha

"tumor necrosis factors/antagonists and inhibitors" [MeSH] OR "anti-TNF" OR "TNF alpha antagonist" OR "tumor necrosis factor inhibitors" $O R$ "tumor necrosis factor antagonist" OR "tumor necrosis factor blocker".

"Remission induction" AND "rheumatoid arthritis" [MeSH].

\section{Sélection des articles}

Nous avons sélectionné les articles rédigés en anglais ou en français concernant des études publiées au cours des dix dernières années (de janvier 2003 à avril 2013). Les revues de la littérature ont été systématiquement retenues et leur bibliographie analysée afin de compléter le recueil des articles. 
Lors de la sélection des études, il a été rapidement mis en évidence que peu d'études cliniques étaient publiées et qu'elles avaient un faible niveau de preuve. Nous avons retenu l'ensemble des articles publiés sur le sujet, à partir des mots clés décrits ci-dessus, même ceux présentant un faible niveau de preuve (un rapport de cas clinique et plusieurs études non contrôlées). Certaines ont toutefois été exclues en cours d'analyse pour les raisons suivantes : description de l'étude incomplète (type d'intervention non spécifié, critères de choix des patients imprécis, variabilité des modalités de gestion des traitements immunosuppresseurs au sein d'une même étude), manque de précisions sur les résultats ou difficulté d'interprétation des résultats (nombreux facteurs de comorbidité, définitions approximatives des critères d'évaluation).

\section{Extraction des données et synthèse}

Les informations concernant les études retenues ont été extraites à l'aide d'une grille d'extraction des données réalisée à cet effet, puis elles ont été synthétisées sous forme de tableaux.

\section{Résultats}

La recherche a répertorié les recommandations de six sociétés savantes dont cinq sociétés de rhumatologie: American College of Rheumatology (ACR), British College of Rheumatology (BCR), Club Rhumatisme et Inflammation (CRI), Japan College of Rheumatology (JCR), Canadian Rheumatology Association (CRA) et de la Société française de chirurgie orale (SFCO).

La plupart des articles retenus étaient des études rétrospectives ou des rapports de cas concernant le suivi de patients ayant subi une chirurgie orthopédique. Un seul rapport de cas concernait directement une complication apparue dans la cavité buccale. Le niveau de preuve de ces études était donc de 3 ou 4 suivant le guide d'analyse de la littérature et de gradation des recommandations de l'HAS [8].

Concernant le risque de complications infectieuses postchirurgicales pour les patients sous anti-TNF alpha, 126 articles ont été identifiés et, après exclusion des articles hors critères, 16 articles ont été retenus. Concernant la cicatrisation et les anti-TNF alpha, 14 articles ont été identifiés et 8 d'entre eux ont été retenus. Enfin, 117 articles concernant le risque de poussée de la PR en cas de suspension du traitement par anti-TNF alpha ont été sélectionnés et 3 ont été retenus. Parmi les articles sélectionnés, toutes les études étaient rétrospectives exceptée celle de Bibbo et Goldberg [9] qui était prospective. La plupart des articles étudiaient les suites postopératoires en chirurgie orthopédique, essentiellement après arthroplastie totale de hanche, chez des patients atteints de PR. Seules quelques études, telles que celle de Ruyssen-Witrand et al. [10] ou de Wendling et al. [14] rapportaient des cas de chirurgie abdominale dans le même contexte.
Au total, 27 articles (certains étudiant à la fois le risque infectieux et les complications cicatricielles) ont été répartis selon les trois axes d'analyse de la problématique et étudiés en détail. Les recommandations de six sociétés scientifiques ont également été analysées.

\section{Évaluation du risque de complications infectieuses postchirurgicales des anti-TNF alpha}

Les études cherchant à évaluer le risque de complications infectieuses en fonction de l'arrêt ou du maintien des anti-TNF avant une intervention chirurgicale ont été répertoriées (Tab. Ia). Plusieurs études ont évalué la fréquence de ces complications postopératoires dans un contexte de chirurgie articulaire [10-17]. Sept études montraient que le maintien des anti-TNF n'a pas entraîné davantage d'infections du site opératoire $[10-15,17]$. En revanche, les infections postopératoires seraient plus sévères chez les patients sous anti-TNF [10, 15]. À l'inverse, dans une étude rétrospective menée en orthopédie chez 91 patients, Giles et al. rapportaient davantage d'infections sévères chez les patients ayant maintenu leur traitement anti-TNF par rapport aux patients sous traitement classique, en excluant les biothérapies [16]. Un cas d'ostéomyélite a été décrit après une avulsion dentaire (sous antibioprophylaxie) chez un patient traité par IFX [18].

Les études cherchant à comparer les pourcentages de complications infectieuses chez les patients sous anti-TNF alpha ayant suspendu leur traitement avant une chirurgie et les patients sous nbDMARDs (non-biologic Disease-Modifying Anti Rheumatic Drugs) tels que le méthotrexate, sulfasalazine et léflunomide ont été répertoriées (Tab. Ib). Les résultats étaient contradictoires. Deux études concluaient à un risque de complications supérieur pour les patients ayant suspendu leur traitement anti-TNF [7, 19]. Quatre études retrouvaient des résultats similaires pour chacun des groupes [9, 20-22].

\section{Impact des anti-TNF sur la cicatrisation}

La majorité des études ont conclu que les anti-TNF ne modifiaient pas la cicatrisation postopératoire $[7,9,11,13$, $14,21,23]$. Une étude a conclu que le délai de cicatrisation pouvait être majoré [10]. Le CRI (Club Rhumatisme et Inflammation) considérait que les complications au cours de la cicatrisation pour les patients sous anti-TNF alpha sont peu probables [24].

\section{Risque de poussée et suspension des anti-TNF alpha}

Aucun article n'avait pour objectif principal d'évaluer le risque de poussée de la polyarthrite rhumatoïde en cas de suspension du traitement par anti-TNF dans le cadre d'une intervention chirurgicale. Quelques observations au cours d'études aux objectifs différents ont été répertoriées. Talwalkar 


\begin{tabular}{|c|c|c|c|c|c|c|c|c|c|}
\hline 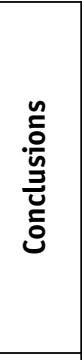 & 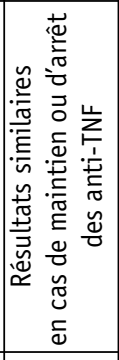 & 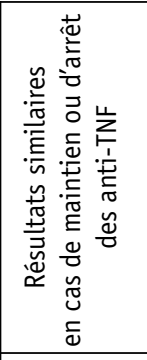 & 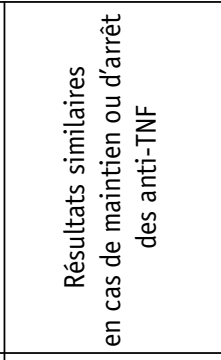 & 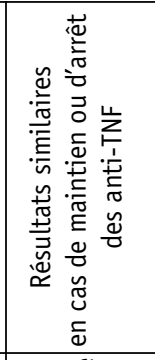 & 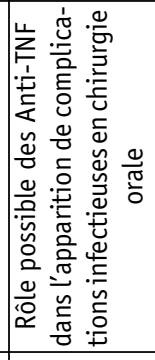 & 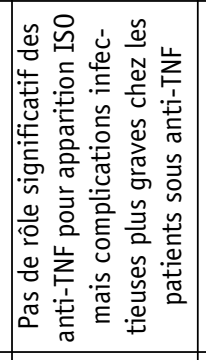 & 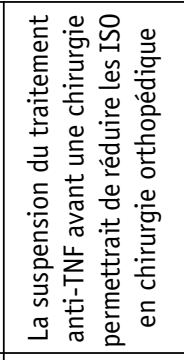 & 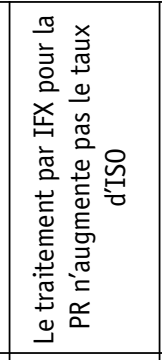 & 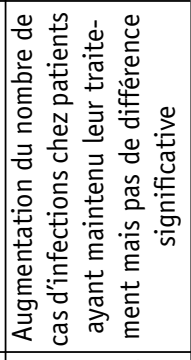 \\
\hline 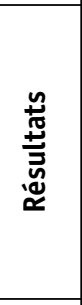 & 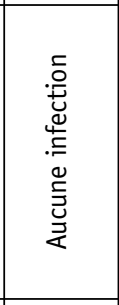 & 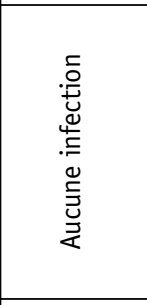 & 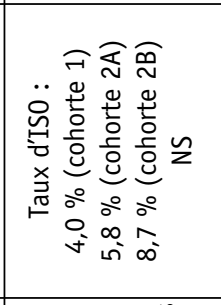 & 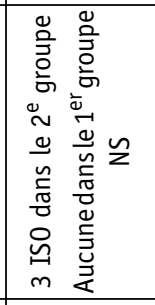 & 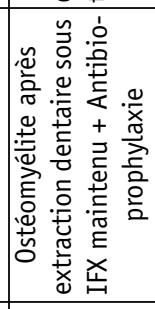 & 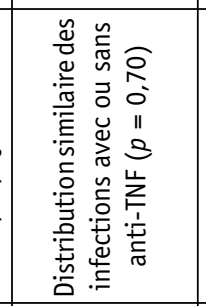 & 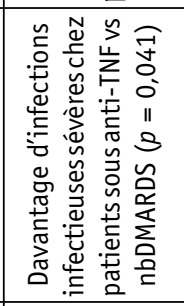 & 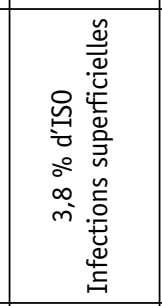 & 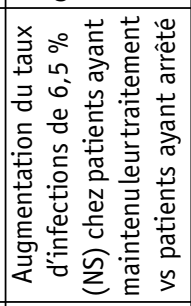 \\
\hline 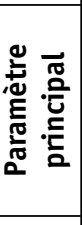 & $\begin{array}{l}\stackrel{\mathscr{G}}{\circ} \\
\stackrel{\circ}{\circ} \\
\circ\end{array}$ & $\begin{array}{l}\stackrel{\stackrel{⿹}{G}}{\circ} \\
\therefore\end{array}$ & 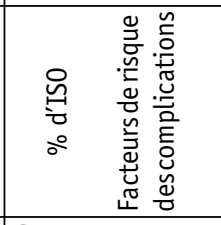 & 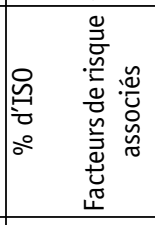 & & 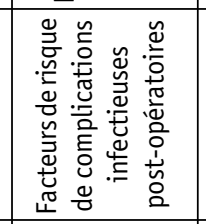 & 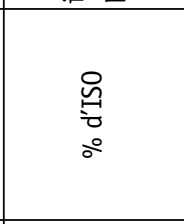 & $\begin{array}{l}0 \\
\ddots \\
\circ\end{array}$ & 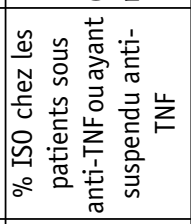 \\
\hline 壳 & 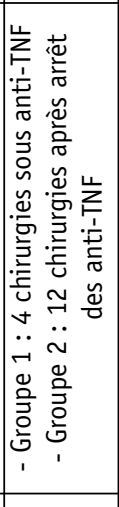 & 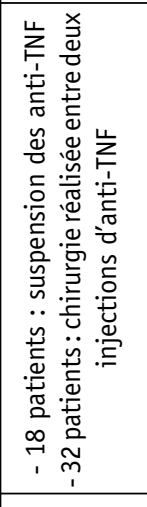 & 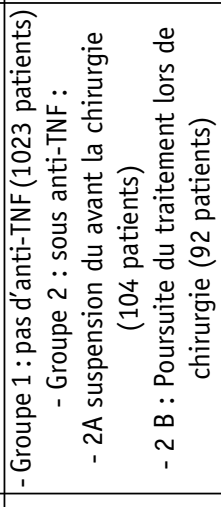 & 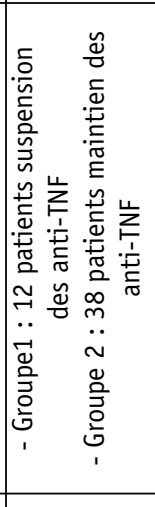 & 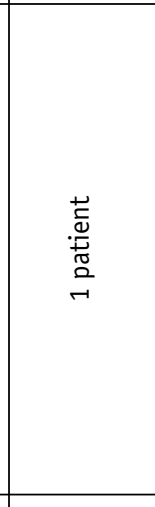 & 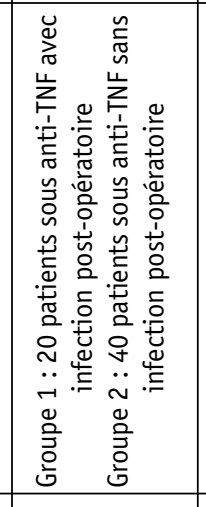 & 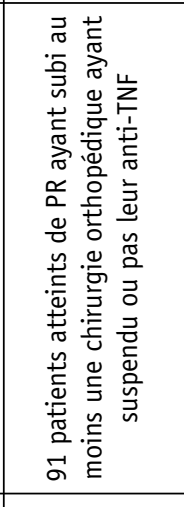 & 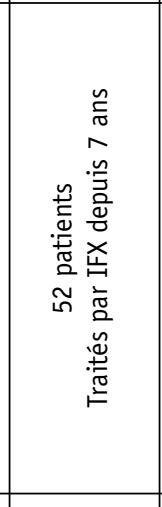 & 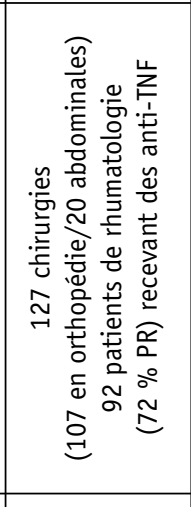 \\
\hline 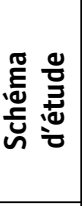 & 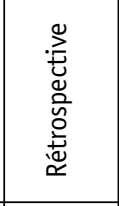 & 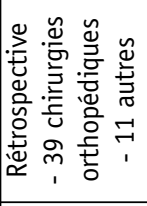 & 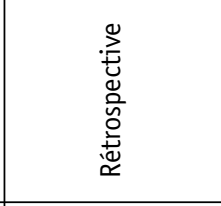 & 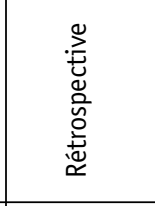 & 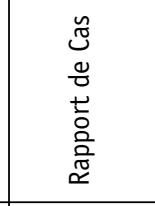 & 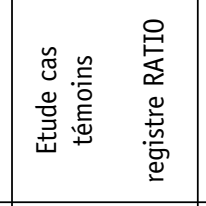 & 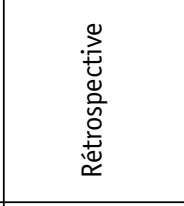 & 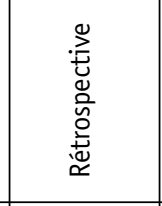 & 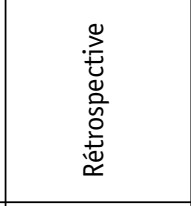 \\
\hline 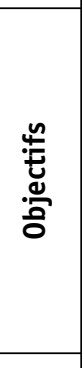 & 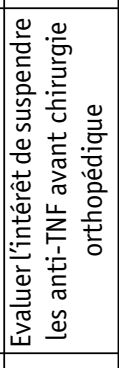 & 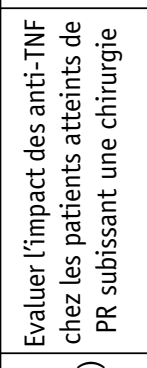 & 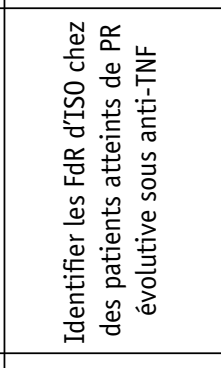 & 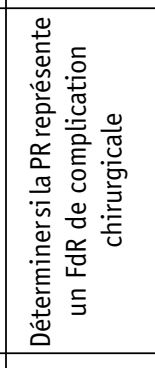 & 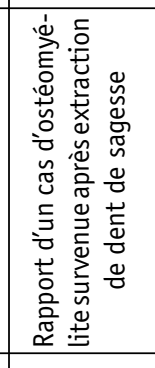 & 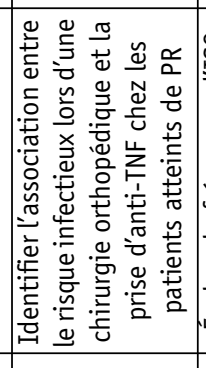 & 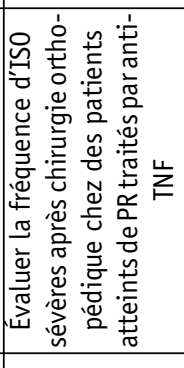 & 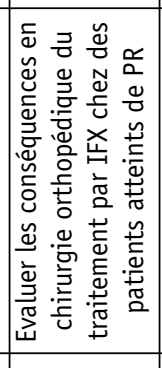 & 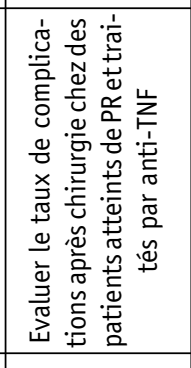 \\
\hline 웜 & $\frac{\propto}{z}$ & 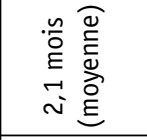 & $\underset{\sim}{\stackrel{c}{\sigma}}$ & $\begin{array}{c}\text { 品 } \\
\infty\end{array}$ & $\begin{array}{l}\stackrel{n}{0} \\
\stackrel{\underline{E}}{m}\end{array}$ & $\frac{\alpha}{z}$ & $\begin{array}{l}\text { ñ } \\
i n\end{array}$ & 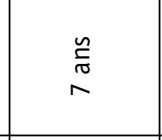 & $\frac{\alpha}{z}$ \\
\hline 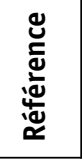 & 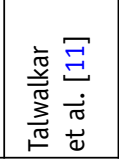 & 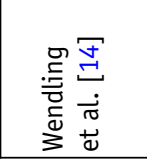 & 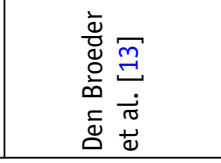 & 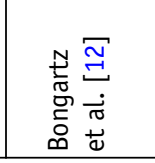 & 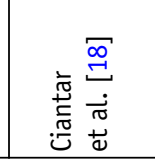 & 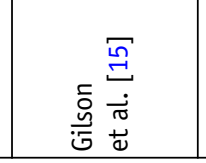 & 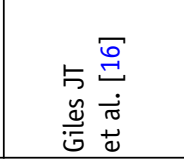 & 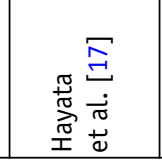 & 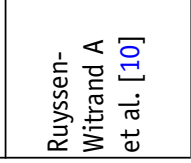 \\
\hline
\end{tabular}




\begin{tabular}{|c|c|c|c|c|c|c|c|}
\hline 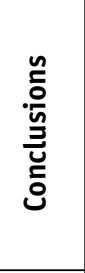 & 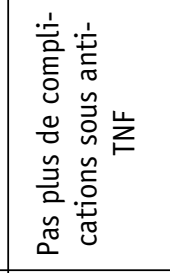 & 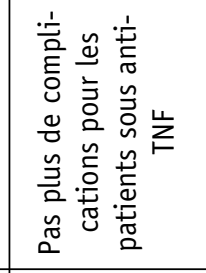 & 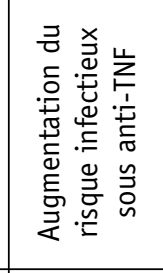 & 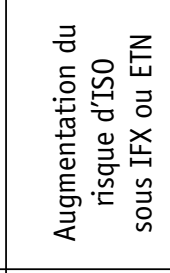 & 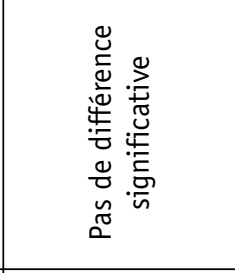 & 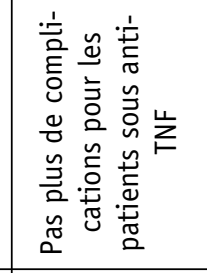 & \\
\hline 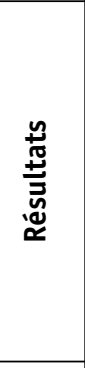 & 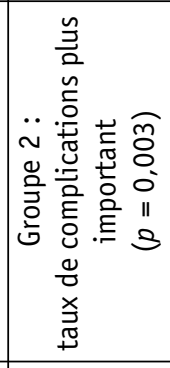 & 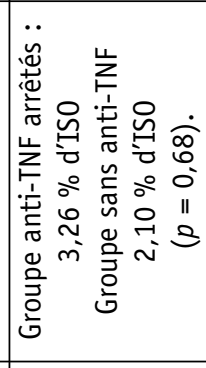 & 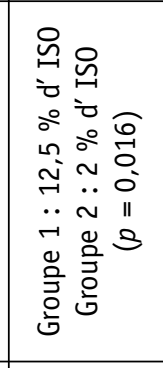 & 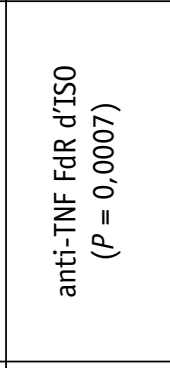 & 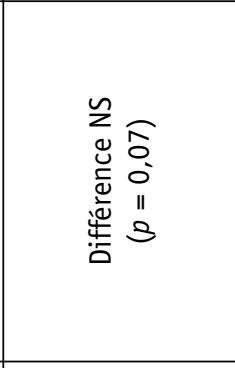 & 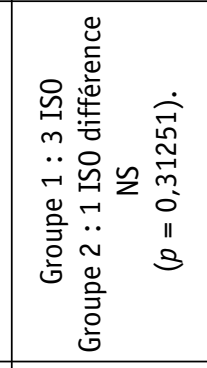 & \\
\hline 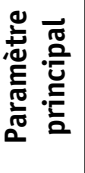 & $\begin{array}{l}\stackrel{\circ}{G} \\
\stackrel{9}{0} \\
\circ\end{array}$ & $\begin{array}{l}\stackrel{\mathscr{G}}{\circ} \\
\stackrel{\circ}{\circ}\end{array}$ & 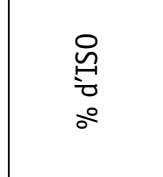 & $\begin{array}{l}\stackrel{9}{\mathscr{H}} \\
\stackrel{0}{\circ} \\
\therefore\end{array}$ & 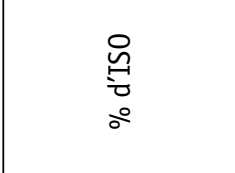 & $\begin{array}{l}\stackrel{9}{G} \\
\stackrel{\circ}{\circ} \\
\circ\end{array}$ & \\
\hline 壱 & 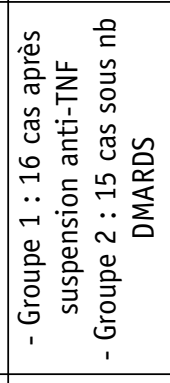 & 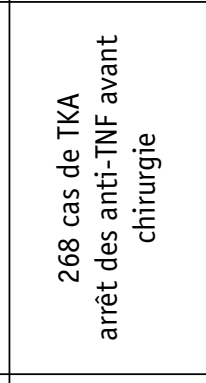 & 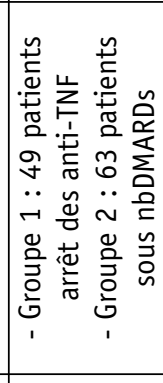 & 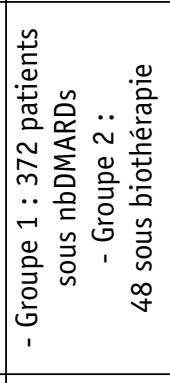 & 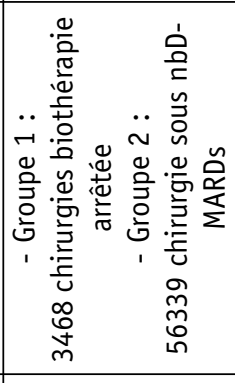 & 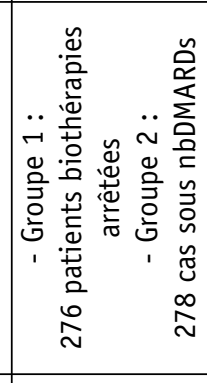 & \\
\hline 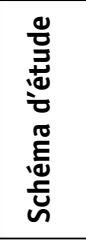 & 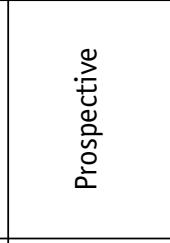 & 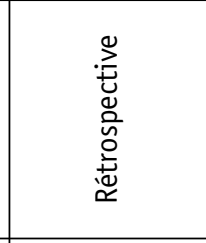 & 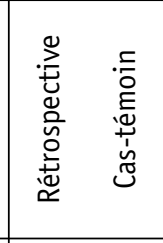 & 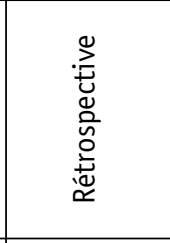 & 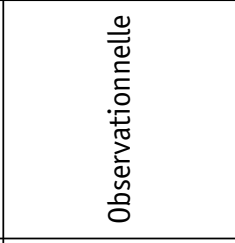 & 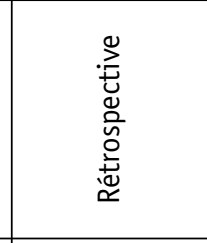 & \\
\hline 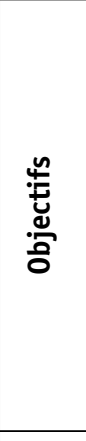 & 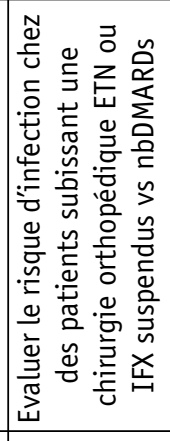 & 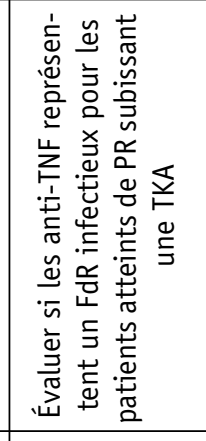 & 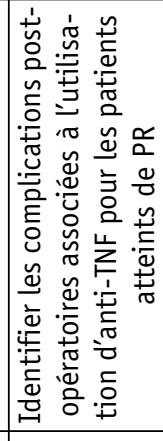 & 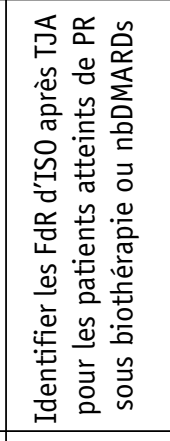 & 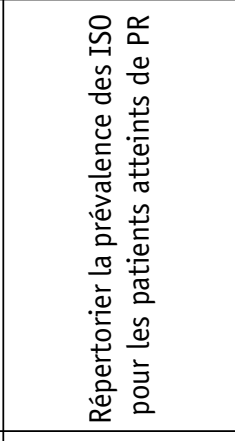 & 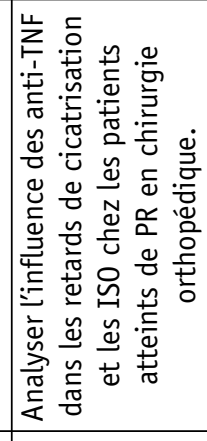 & \\
\hline 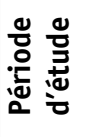 & $\begin{array}{l}\stackrel{n}{O} \\
\stackrel{E}{\simeq} \\
\cong\end{array}$ & $\begin{array}{l}\frac{n}{0} \\
\frac{E}{6}\end{array}$ & $\frac{\alpha}{z}$ & $\frac{\alpha}{z}$ & $\frac{\alpha}{z}$ & $\frac{\alpha}{z}$ & \\
\hline & 㐫 & 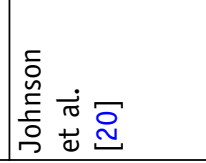 & 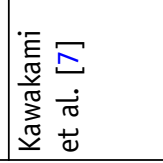 & 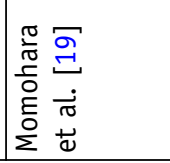 & 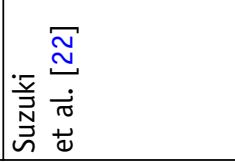 & 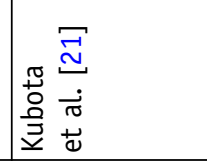 & \\
\hline
\end{tabular}


et al. [11] rapportaient un cas de poussée alors que l'ETN avait été suspendu chez un patient. Wendling [14] a rapporté un taux de poussée de $12 \%$ associé à la suspension de l'ETN avant des chirurgies. Kawakami rapportait un taux de poussée de 17,2 \% associé à la suspension de l'ETN avant des chirurgies [7].

\section{Discussion}

L'objectif de cette étude était d'évaluer le rapport bénéficerisque de la suspension des anti-TNF alpha avant une chirurgie orale. Il s'agissait d'une analyse systématique de la littérature entre 2003 et 2013. Le rapport bénéfice-risque a été évalué selon trois critères : l'influence des anti-TNF sur la survenue d'infections, l'influence des anti-TNF sur la cicatrisation et l'influence de la suspension des anti-TNF sur le risque de poussée de PR.

Il faut souligner qu'aucune étude contrôlée n'a été publiée en chirurgie orale ou maxillo-faciale. Seul un rapport de cas d'ostéite mandibulaire survenue après une extraction dentaire chez un patient traité par IFX, maintenu lors de l'intervention, a été décrit [18]. Plus récemment, un cas d'ostéonécrose mandibulaire a été observé chez une patiente de 36 ans traitée pour une maladie de Crohn par corticostéroïdes au long cours [10 ans] ainsi que par risédronate, zolédronate et adalimumab depuis deux ans. Dans ce cas, il est difficile d'isoler un seul traitement causal, mais les auteurs rapportent la possible implication des anti-TNF [25]. En réalité, l'imputabilité des biphosphonates associés aux corticoïdes semble plus importante que celle des anti-TNF.

La revue de la littérature a été faite sur des études d'évaluation du risque infectieux secondaire à la pose d'une prothèse orthopédique. Cela représente un biais pour tirer des conclusions en chirurgie orale. Cependant, le risque infectieux postopératoire peut être estimé par l'indice de risque NNIS (National Nosocomial Infection Surveillance) qui prend en compte la classification d'Altemeier, la durée de l'intervention et le score ASA [26]. En l'absence de PR, la majorité des interventions de chirurgie orale ont un score NNIS de 0 ou 1, ce qui est identique à la chirurgie orthopédique. En l'absence de PR, les taux d'infection postopératoires pour l'extraction d'une dent de sagesse vont de 0,9 à 5,9\% [27]. Ils sont similaires à ceux de la chirurgie articulaire (2 à $5 \%$ ) [28, 29]. En présence de PR, la majorité des études n'a pas identifié davantage de complications infectieuses postopératoires en cas de maintien des anti-TNF par rapport aux groupes les ayant suspendu [10-15]. La majorité des études n'a pas retrouvé plus de complications postopératoires entre le groupe ayant suspendu son traitement par anti-TNF et le groupe témoin sous nbDMARDs [9, 20-22]. En revanche, il est difficile de conclure car les études sont essentiellement rétrospectives, les échantillons sont souvent réduits, il manque des informations sur la nature des complications, l'éventuelle prescription d'antibioprophylaxie en préopératoire, les cofacteurs de morbidité ou encore la durée de suspension variable des anti-TNF. On sait que les anti-TNF antagonisent la fonction antibactérienne du TNF alpha et il a été prouvé que, peu de temps après l'instauration de ce traitement, une neutropénie apparaissait et se maintenait quelques semaines [30]. Finalement, le véritable enjeu est de déterminer si ces médicaments engendrent un risque d'infection sévère majoré. $0 r$, l'association entre infection sévère et anti-TNF alpha n'est pas clairement établie. Pour le registre britannique [31], les patients sous anti-TNF alpha auraient un taux d'infections sévères plus élevé, le hasard-ratio ajusté pour les infections sévères était $20 \%$ plus élevé dans la cohorte sous anti-TNF alpha par rapport au groupe sous nbDMARDs (adj HR : $1,2$ [IC $95 \%, 1,1-1,5]$ ). Le risque ne semblait pas différer significativement entre l'infliximab, l'étanercept et l'adalimumab. Le registre allemand [32] et l'étude d'Atzeni et al. [33] ont cependant mis en évidence que l'ETN engendrait moins de complications infectieuses. D'après les registres CORRONA [34] et Grijalva et al. [35], la corticothérapie (sans que soient précisées ni la molécule ni la dose) favoriserait l'apparition d'infections sévères alors que les anti-TNF et les nbDMARDs ne seraient pas impliqués. Schneeweiss et al. [36] en 2007 n'ont pas retrouvé davantage de risque d'infections sévères parmi 15597 patients atteints de PR après ajustement des données entre la prise d'anti-TNF et la prise de MTX (RR 1,0 [95\% CI $0,6-1,7])$. La méta-analyse de Leombruno et al. [37] n'a pas non plus mis en évidence de différence significative entre les patients sous anti-TNF et les patients sous nbDMARDs. Plusieurs études $[31,38,39]$ ont conclu à une augmentation du risque infectieux au cours des six premiers mois de traitement, le temps que la maladie inflammatoire soit stabilisée. La PR représente un risque inhérent d'infection imputé au dysfonctionnement du système immunitaire. L'étude rétrospective longitudinale de Doran et al. [40] a montré un risque relatif de $1,53(95 \%$ CI 1,41-1,65) de développer une infection postopératoire pour un patient atteint de $\mathrm{PR}$, en comparaison avec un sujet témoin. Dans ce contexte, seule l'utilisation de MTX au cours de chirurgies a été suffisamment étudiée pour pouvoir conclure que son emploi n'augmentait pas le taux de complications infectieuses postopératoires et qu'il peut être maintenu en peropératoire [41]. Des études ont établi l'existence de cofacteurs de risque d'infection pour les patients sous antiTNF atteints de PR. Il s'agit de la prise de corticoïdes [7, 42], la durée d'évolution de la PR [7, 19], les antécédents d'infection du site opératoire [13], un âge avancé à la mise en place d'un traitement de fond [33] et l'existence de comorbidités (BCP0, alcoolisme, diabète sucré) [40]. Il n'est pas possible de conclure sur le risque infectieux postopératoire des patients sous anti-TNF car les études sont essentiellement rétrospectives, les échantillons sont différents selon les études et certains résultats sont contradictoires. Des études prospectives devraient être conduites afin de déterminer le risque infectieux postopératoire des patients sous anti-TNF, comme cela a été fait pour le MTX. 


\begin{tabular}{|c|c|c|c|c|c|c|c|c|}
\hline 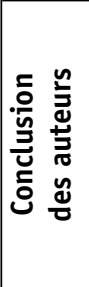 & 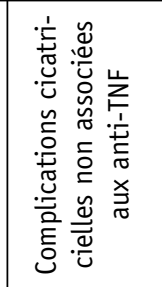 & 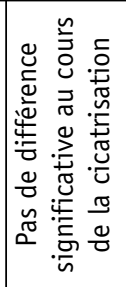 & 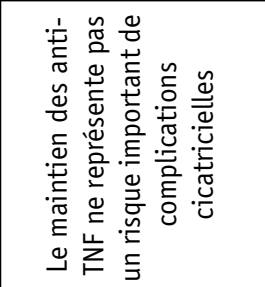 & 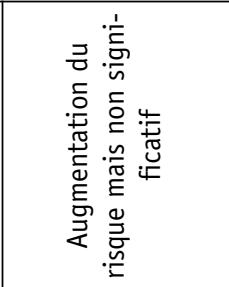 & 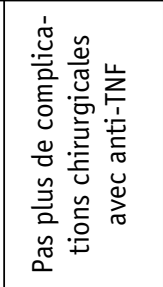 & 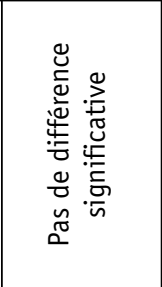 & 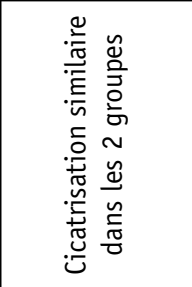 & 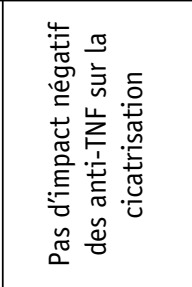 \\
\hline 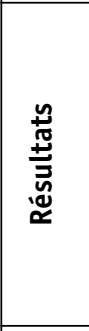 & 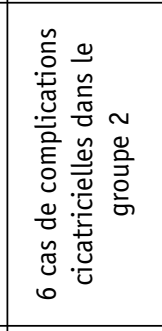 & 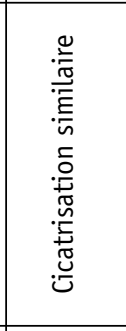 & 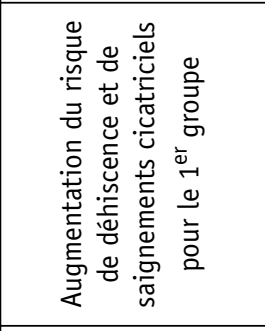 & 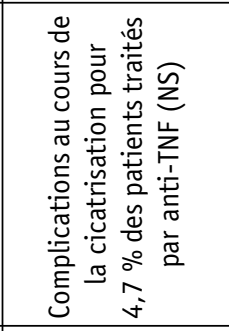 & 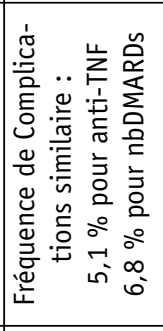 & 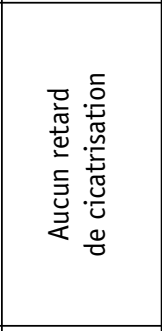 & 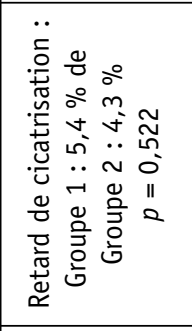 & 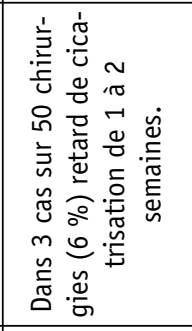 \\
\hline 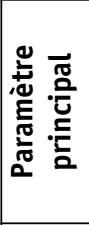 & 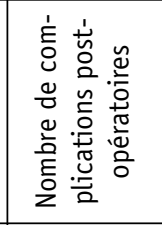 & $\begin{array}{l}\stackrel{\circ}{G} \\
\text { 。 } \\
\text { 。 }\end{array}$ & 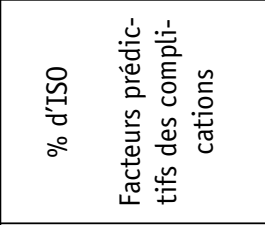 & $\begin{array}{l}\stackrel{\mathscr{G}}{\circ} \\
\circ\end{array}$ & 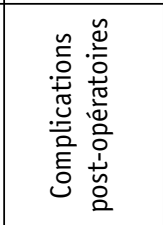 & $\begin{array}{l}\stackrel{\bigcirc}{G} \\
\stackrel{6}{\circ} \\
\therefore\end{array}$ & $\begin{array}{l}\stackrel{९}{\mathscr{H}} \\
\stackrel{0}{0} \\
\circ\end{array}$ & $\begin{array}{l}\stackrel{ }{\mathscr{H}} \\
\stackrel{0}{\circ} \\
\circ\end{array}$ \\
\hline 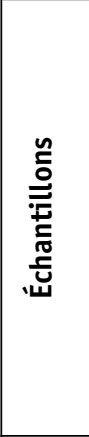 & 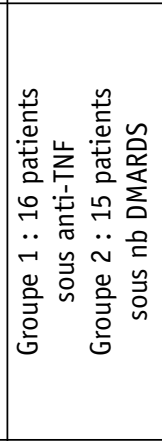 & 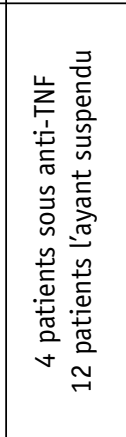 & 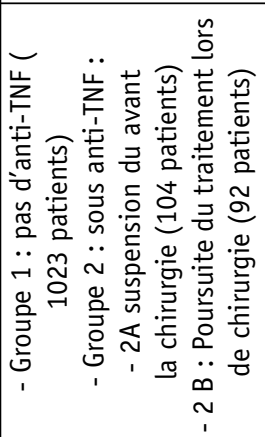 & 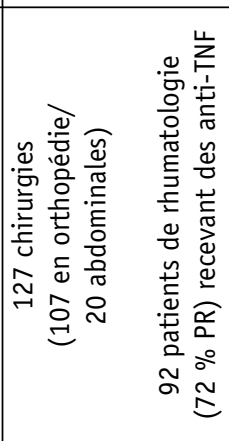 & 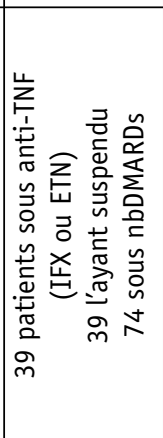 & 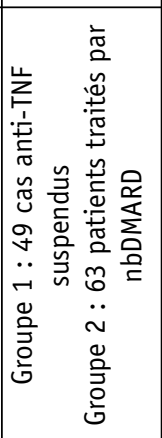 & 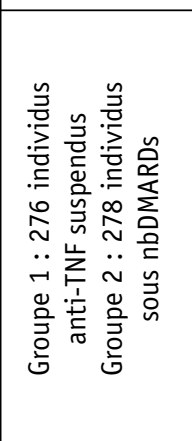 & 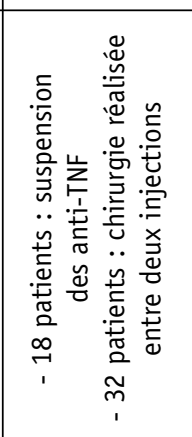 \\
\hline 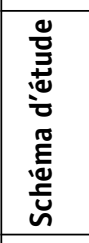 & 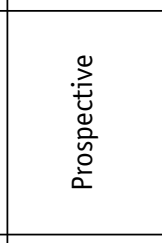 & 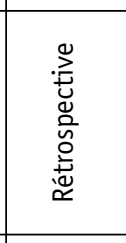 & 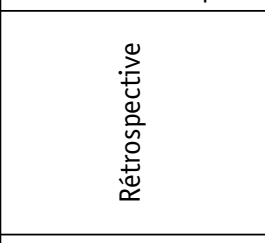 & 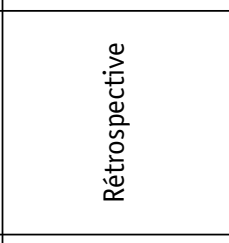 & 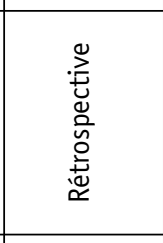 & 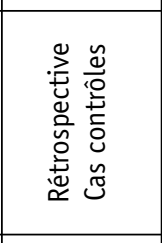 & 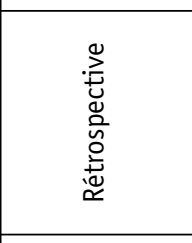 & 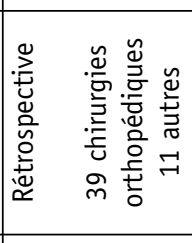 \\
\hline 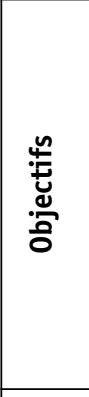 & 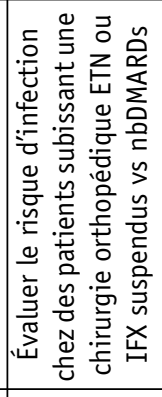 & 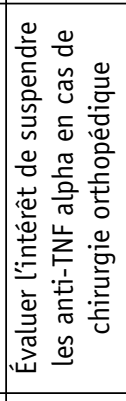 & 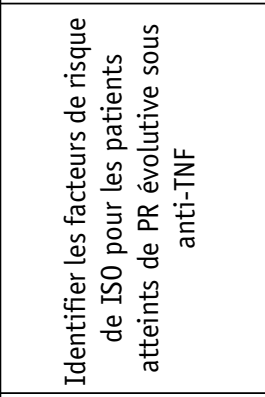 & 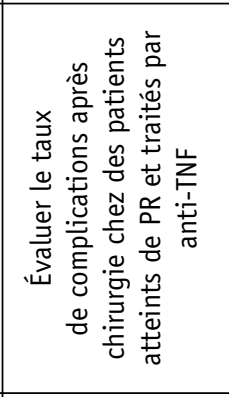 & 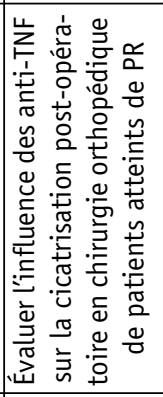 & 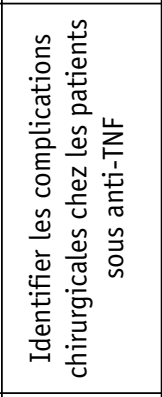 & 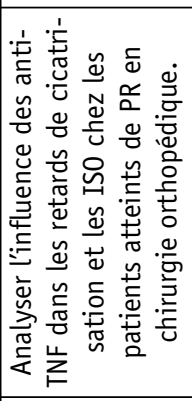 & 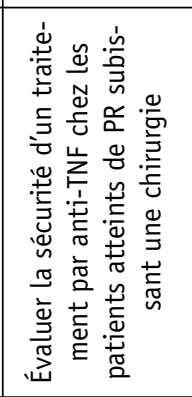 \\
\hline 苋 & 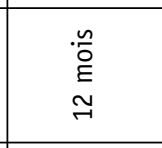 & $\frac{\mathscr{c}}{z}$ & $\underset{\sim}{\stackrel{c}{\sigma}}$ & 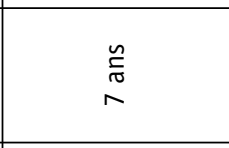 & 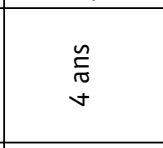 & 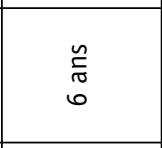 & $\frac{\underline{c}}{z}$ & 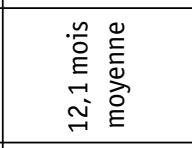 \\
\hline 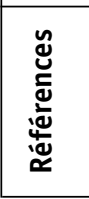 & 完 & 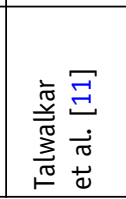 & 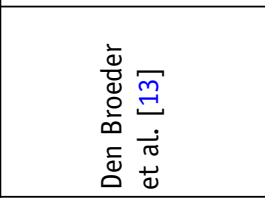 & 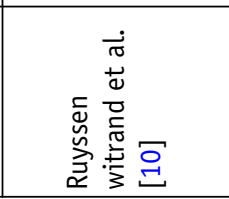 & 苞 & 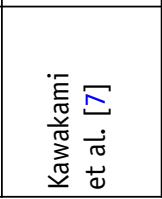 & 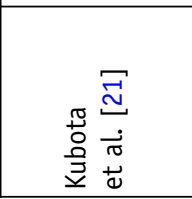 & 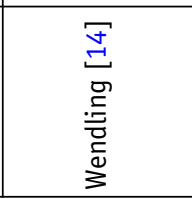 \\
\hline
\end{tabular}


En cas d'intervention chirurgicale, les patients atteints de PR pourraient présenter un retard de cicatrisation et les patients sous anti-TNF auraient un risque majoré de complications cicatricielles [43]. En effet, le TNF alpha est essentiel au bon déroulement de la cicatrisation. Dans une étude chez la souris sous ETN, Iglesias et al. ont conclu à une durée de cicatrisation similaire avec ou sans anti-TNF [44]. Plusieurs études rétrospectives [11-14] ainsi que l'étude prospective de Bibbo et al. [9] n'ont pas conclu à davantage de complications chez les patients sous anti-TNF. La majorité des données récentes sont rassurantes mais des études prospectives sur de larges cohortes sont nécessaires pour établir une recommandation fondée sur la preuve.

La complication possible de l'arrêt des anti-TNF avant une chirurgie est un risque de poussée de la pathologie chronique. Une poussée correspond à une phase d'évolution de la maladie. Pour prendre en charge cette poussée, le patient reçoit souvent une dose importante de corticoïdes, ce qui peut avoir pour conséquence d'augmenter le risque infectieux et de retarder la cicatrisation. Un cas de poussée a été rapporté lors d'un arrêt de l'ETN [11]. La reprise du traitement a permis de contrôler cette poussée, sans complications. Des fréquences de poussées de 12 à $17,2 \%$ ont été associées significativement à la suspension de l'ETN [7, 14], alors que l'arrêt de l'IFX entraînait rarement des poussées de la PR. Ces résultats pourraient s'expliquer par la demi-vie plus longue d'IFX (10 jours versus $70 \mathrm{~h}$ pour ETN). De plus, l'action de l'IFX sur la cytokine est basée sur une liaison irréversible antigène-anticorps alors que l'ETN est un récepteur qui se lie de façon réversible à la molécule de TNF alpha [7,23]. Après avoir obtenu un faible niveau d'activité de la maladie suite à un traitement intensif par antiTNF, il est possible de constater une période de rémission sans poussée de la PR [23, 45-47]. Les patients diagnostiqués et pris en charge précocement semblent plus enclins à rester en rémission que les patients ayant une pathologie établie $[23,46]$. Il n'est pas possible de conclure définitivement sur le risque de poussée de PR après suspension du traitement antiTNF, mais les résultats disponibles semblent montrer qu'il serait plus important avec des anti-TNF de demi-vie courte [7].

\section{Conclusion}

Du fait du manque d'études contrôlées en chirurgie orale, il n'est pas possible de conclure définitivement sur l'intérêt de suspendre un traitement anti-TNF alpha chez un patient atteint de PR, afin de prévenir une infection ou pour favoriser la cicatrisation. Pour la même raison, il n'est pas possible de conclure sur le risque de poussée de la PR après suspension des antiTNF alpha.

Cependant, même si les études citées précédemment n'ont pas un niveau de preuve suffisant, elles ne semblent pas mettre en évidence de majoration du risque infectieux, ni de retard de cicatrisation. Ainsi, il semble intéressant de proposer une attitude moins systématique d'arrêt des anti-TNF en chirurgie orale. La décision devra être prise en considérant les facteurs susceptibles de favoriser l'évolution de la PR lors d'un arrêt des anti-TNF alpha (ancienneté de la PR, évolution, durée de la demi-vie des anti-TNF alpha), ainsi que les facteurs susceptibles de favoriser l'infection (classe d'Altemeier, score ASA, durée d'intervention).

\section{Conflits d'intérêt : aucun}

\section{Références}

1. Smolen JS, Aletaha D, Koeller M, Weisman MH, Emery P. New therapies for treatment of rheumatoid arthritis. Lancet 2007; 370:1861-74.

2. bio-therapy.net - Recommandations de l'EULAR pour la prise en charge thérapeutique de la polyarthrite rhumatoïde [Internet]. http://www.bio-therapy.net/content/view/3577/229/

3. Kaur S, White $S$, Bartold PM. Periodontal disease and rheumatoid arthritis: a systematic review. J Dent Res 2013;92:399-408.

4. Monsarrat $P$, et al. Oral Health Status in Outpatients with Rheumatoid Arthritis: The OSARA Study. Oral Health Dent Manag 2014;13:113-9.

5. Blaizot A, et al. Oral health-related quality of life among outpatients with rheumatoid arthritis. Int Dent J 2013;63: 45-53.

6. Société française de chirurgie orale [Internet]. [http://www. societechirorale.com/Recommandations/Recommandations.html

7. Kawakami K, et al. Complications and features after joint surgery in rheumatoid arthritis patients treated with tumour necrosis factor-alpha blockers: perioperative interruption of tumour necrosis factor-alpha blockers decreases complications? Rheumatology 2010;49:341-7.

8. Agence nationale d'accréditation et d'évaluation en santé. Guide d'analyse de la littérature et gradation des recommandations [Internet]. 2000 http://www.has-sante.fr/portail/upload/docs/ application/pdf/analiterat.pdf

9. Bibbo C, Goldberg JW. Infectious and healing complications after elective orthopaedic foot and ankle surgery during tumor necrosis factor-alpha inhibition therapy. Foot Ankle Int 2004;25:331-5.

10. Ruyssen-Witrand $A$, et al. Complication rates of 127 surgical procedures performed in rheumatic patients receiving tumor necrosis factor alpha blockers. Clin Exp Rheumatol 2007;25:430-6.

11. Talwalkar SC, Grennan DM, Gray J, Johnson P, Hayton MJ. Tumour necrosis factor alpha antagonists and early postoperative complications in patients with inflammatory joint disease undergoing elective orthopaedic surgery. Ann Rheum Dis 2005;64:650-1.

12. Bongartz $T$, et al. Incidence and risk factors of prosthetic joint infection after total hip or knee replacement in patients with rheumatoid arthritis. Arthritis Rheum 2008;59:1713-20.

13. den Broeder AA, et al. Risk factors for surgical site infections and other complications in elective surgery in patients with rheumatoid arthritis with special attention for anti-tumor necrosis factor: a large retrospective study. J Rheumatol 2007; 34:689-95.

14. Wendling $D$, et al. Surgery in patients receiving anti-tumour necrosis factor alpha treatment in rheumatoid arthritis: an observational study on 50 surgical procedures. Ann Rheum Dis 2005;64:1378-9. 
15. Gilson M, et al. Risk factors for total joint arthroplasty infection in patients receiving tumor necrosis factor -blockers: a casecontrol study. Arthritis Res Ther 2010;12:R145.

16. Giles JT, et al. Tumor necrosis factor inhibitor therapy and risk of serious postoperative orthopedic infection in rheumatoid arthritis. Arthritis Rheum 2006;55:333-7.

17. Hayata K, Kanbe K, Chiba J, Nakamura A, Inoue Y, Hobo K. Clinical factors related to the efficacy and complications of orthopedic surgery for rheumatoid arthritis with infliximab. Int $\mathrm{J}$ Rheum Dis 2011;14:31-6.

18. Ciantar M, Adlam DM. Treatment with infliximab: Implications in oral surgery? A case report. Br J Oral Maxillofac Surg 2007; 45:507-10.

19. Momohara S, et al. Prosthetic joint infection after total hip or knee arthroplasty in rheumatoid arthritis patients treated with nonbiologic and biologic disease-modifying antirheumatic drugs. Mod Rheumatol 2011;21:469-75.

20. Johnson BK, Goodman SM, Alexiades MM, Figgie MP, Demmer RT, Mandl LA. Patterns and associated risk of perioperative use of antitumor necrosis factor in patients with rheumatoid arthritis undergoing total knee replacement. J Rheumatol 2013;40:617-23.

21. Kubota A, Nakamura T, Miyazaki Y, Sekiguchi M, Suguro T. Perioperative complications in elective surgery in patients with rheumatoid arthritis treated with biologics. Mod Rheumatol 2012;22:844-8.

22. Suzuki M, Nishida K, Soen S, Oda H, Inoue H, Kaneko A, et al. Risk of postoperative complications in rheumatoid arthritis relevant to treatment with biologic agents: a report from the Committee on Arthritis of the Japanese Orthopaedic Association. J Orthop Sci 2011;16:778-84.

23. Hirano $Y$, et al. Influences of anti-tumour necrosis factor agents on postoperative recovery in patients with rheumatoid arthritis. Clin Rheumatol 2010;29:495-500.

24. Bienvenue sur le Club Rhumatismes et Inflammations [Internet]. http://www.cri-net.com/recherche/fichespratiques/ recommandations.asp

25. Preidl RH, Ebker T, Raithel M, Wehrhan F, Neukam FW, Stockmann P. Osteonecrosis of the jaw in a Crohn's disease patient following a course of Bisphosphonate and Adalimumab therapy: a case report. BMC Gastroenterol 2014;14:6.

26. Emori TG, et al. National nosocomial infections surveillance system [NNIS]: description of surveillance methods. Am J Infect Control 1991;19:19-35.

27. Blondeau F, Daniel NG. Extraction of impacted mandibular third molars: postoperative complications and their risk factors. J Can Dent Assoc 2007;73:325.

28. Chadli $M$, et al. [Incidence of surgical wound infections a prospective study in the Rabat Mohamed-V military hospital, Morocco]. Med Mal Infect 2005;35:218-22.

29. Gaynes R, et al. Feeding back surveillance data to prevent hospital-acquired infections. Emerg Infect Dis 2001;7:295-8.

30. Vidal F, Fontova R, Richart C. Severe neutropenia and thrombocytopenia associated with infliximab. Ann Intern Med 2003; 139:W-W63.

31. Ding $T$, et al. BSR and BHPR rheumatoid arthritis guidelines on safety of anti-TNF therapies. Rheumatology 2010;49:2217-9.
32. Van Dartel SAA, et al. Difference in the risk of serious infections in patients with rheumatoid arthritis treated with adalimumab, infliximab and etanercept: results from the Dutch Rheumatoid Arthritis Monitoring [DREAM] registry. Ann Rheum Dis 2012; 72:895-900.

33. Atzeni $F$, et al. Long-term anti-TNF therapy and the risk of serious infections in a cohort of patients with rheumatoid arthritis: comparison of adalimumab, etanercept and infliximab in the GISEA registry. Autoimmun Rev 2012;12:225-9.

34. Kremer J. The CORRONA database. Ann Rheum Dis 2005;64: iv37-iv41.

35. Grijalva CG, Kaltenbach L, Arbogast PG, Mitchel EF Jr, Griffin MR. Initiation of rheumatoid arthritis treatments and the risk of serious infections. Rheumatology 2010;49:82-90.

36. Schneeweiss $S$, et al. Anti-tumor necrosis factor therapy and the risk of serious bacterial infections in elderly patients with rheumatoid arthritis. Arthritis Rheum 2007;56:1754-64.

37. Leombruno JP, Einarson TR, Keystone EC. The safety of antitumour necrosis factor treatments in rheumatoid arthritis: meta and exposure-adjusted pooled analyses of serious adverse events. Ann Rheum Dis 2008;68:1136-45.

38. Curtis JR, et al. Risk of serious bacterial infections among rheumatoid arthritis patients exposed to tumor necrosis factor $\alpha$ antagonists. Arthritis Rheum 2007;56:1125-33.

39. Nam JL, et al. Current evidence for the management of rheumatoid arthritis with biological disease-modifying antirheumatic drugs: a systematic literature review informing the EULAR recommendations for the management of RA. Ann Rheum Dis 2010;69:976-86.

40. Doran MF, Crowson CS, Pond GR, O'Fallon WM, Gabriel SE. Predictors of infection in rheumatoid arthritis. Arthritis Rheum 2002;46:2294-300.

41. Grennan DM, Gray J, Loudon J, Fear S. Methotrexate and early postoperative complications in patients with rheumatoid arthritis undergoing elective orthopaedic surgery. Ann Rheum Dis 2001;60:214-7.

42. Greenberg JD, et al. A comparative effectiveness study of adalimumab, etanercept and infliximab in biologically naive and switched rheumatoid arthritis patients: results from the US CORRONA registry. Ann Rheum Dis 2012;71:1134-42.

43. Goh L, Jewell T, Laversuch C, Samanta A. Should anti-TNF therapy be discontinued in rheumatoid arthritis patients undergoing elective orthopaedic surgery? A systematic review of the evidence. Rheumatol Int 2011;32:5-13.

44. Iglesias E, O’Valle F, Salvatierra J, Aneiros-Fernández J, CanteroHinojosa J, Hernández-Cortés P. Effect of blockade of tumor necrosis factor-alpha with etanercept on surgical wound healing in SWISS-OF1 mice. J Rheumatol 2009;36:2144-8.

45. Klarenbeek NB, et al. Discontinuing treatment in patients with rheumatoid arthritis in sustained clinical remission: exploratory analyses from the BeSt study. Ann Rheum Dis 2011;70:315-9.

46. Tanaka $Y$, et al. Discontinuation of infliximab after attaining low disease activity in patients with rheumatoid arthritis: RRR [remission induction by Remicade in RA] study. Ann Rheum Dis 2010;69:1286-91.

47. Brocq 0, et al. Effect of discontinuing TNFalpha antagonist therapy in patients with remission of rheumatoid arthritis. Joint Bone Spine 2009;76:350-5. 